\title{
Metamaterials: Optical Activity Without Chirality
}

\author{
E. Plum, V. A. Fedotov, and N. I. Zheludev* \\ Optoelectronics Research Centre, University of Southampton, SO17 1BJ, UK
}

(Dated: July 28, 2008)

\begin{abstract}
We report that the classical phenomenon of optical activity, which is traditionally associated with chirality (helicity) of organic molecules, proteins and inorganic structures, can be observed in artificial media which exhibit neither volume nor planar chirality (helicity). We have designed a metamaterial film periodically structured on the subwavelength scale which behaves indistinguishably from chiral three-dimensional media yielding strong circular dichroism and birefringence in transmission for waves incident at oblique angles.
\end{abstract}

The phenomenon of optical activity that is the ability to rotate the polarization state of light is a fundamental effect of electrodynamics which is traditionally associated with mirror asymmetry (chirality) of organic molecules. The effect has enormous importance for analytical chemistry, crystallography, molecular biology, and the food industry and is also a signature effect used to detect life forms in space missions. The recognition of chirality as a source negative refraction of light [1-6] needed for the creation of a perfect lens [7] inspired intense work in developing microwave and optical artificial chiral metamaterials [8-12] and yielded a demonstration of negative index due to chirality $[8,13]$. In this paper we present a somewhat surprising result that very strong optical activity may be seen in a metamaterial system consisting of meta-molecules that itself are not chiral. Here chirality is drawn from the mutual orientation of the wave propagation direction and the two-dimensional metamaterial. We demonstrate the optical activity effect using an artificially created non-chiral planar metamaterial structure and show that it behaves indistinguishably from chiral three-dimensional molecular systems manifesting resonant birefringence and dichroism for circularly polarized electromagnetic waves.

Through the efforts of several generations of researchers optical activity was linked to the threedimensional property of molecules known as chirality: a molecular structure such as a helix for which mirror images are not congruent possesses a sufficient asymmetry to manifest polarization rotation (optical activity). The effect of optical activity is linked to the phenomenon of circular dichroism, i.e. differential absorbtion for left and right circular polarizations. The recent effort in creating artificial optically active metamaterials that aimed to achieve strong optical activity was focused on different types of arrays of 3D-chiral meta-molecules [8-12, 14-17]. It is significantly less acknowledged that optical activity can also be seen when oriented non-chiral molecules make a chiral triad with the wave vector of light. This mechanism of optical activity was first described by Bunn [18] and detected in liquid crystals [19]. Here we show that this is a highly significant mechanism of optical activity in metamaterials that can be seen as essentially planar structures that possess neither $2 \mathrm{D}$ chirality $[20,21]$ nor

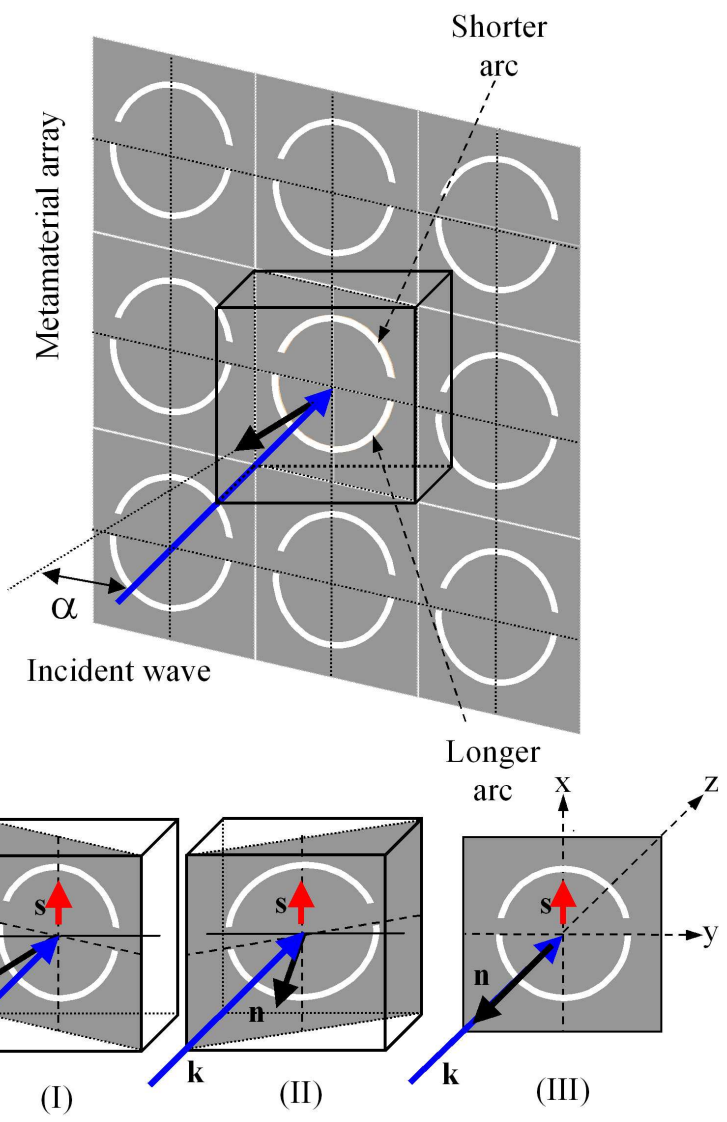

FIG. 1: (Color online) Planar slit metamaterial based on an array of asymmetrically split rings that manifest optical activity and circular dichroism at oblique incidence of light. The direction of asymmetry is represented by a polar vector $s$ (directed from the long to the short arc). Optical activity is seen when the metamaterial plane is tilted around $x$-axis so the sample normal $n$ is at an angle $\alpha \neq 0$ with the wave vector of the incident wave $k$. Configuration I and II are the two enantiomeric arrangements showing optical activity of opposite signs. Configuration III corresponding to normal incidence shows no optical activity.

3D chirality, and which are much simpler to fabricate than metamaterials based on arrays of 3D-chiral metamolecules.

We argue that to manifest optical activity meta- 


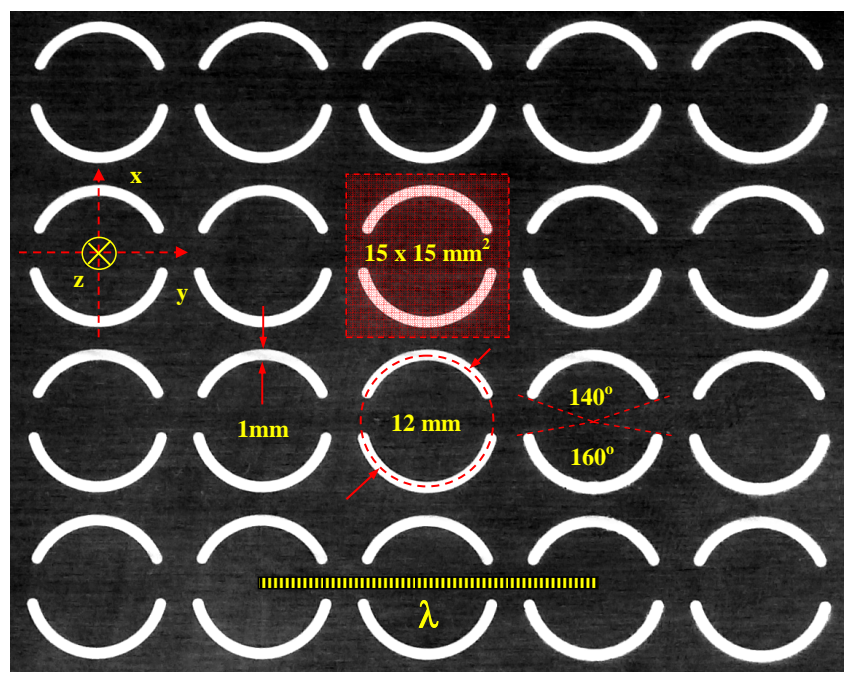

FIG. 2: (Color online) A fragment of the planar slit metamaterial fabricated in $1 \mathrm{~mm}$ thick aluminium film photographed against a light background. The highlighted square section shows the elementary building block of the two-dimensional periodic metamaterial. A bar in the lower part of the image represents the wavelength $\lambda$ at which the polarization resonance was observed.

molecules of a planar metamaterial structure may have a line of mirror symmetry, but shall lack an inversion center, i.e. they shall posses a polar direction $s$ (as illustrated in Fig. 1). A regular oriented array of such metamolecules will not show optical activity at normal incidence. However, the metamaterial will become optically active at oblique incidence provided that the plane of incidence does not contain the polar direction. Indeed, in this case the wave vector $k$, normal to the meta-molecule plane $n$ and polar vector $s$ constitute a three-dimensional chiral triad. The enantiomeric configurations of these vectors corresponding to optical activity of opposite signs are created by tilting the plane of the structure in opposite directions with respect to the incident wave vector (compare I and II in Fig. 1).

The origin of the effect in such a planar non-chiral structure may be readily seen by considering a "unit cell", which contains a section of tilted metal plate with a single split ring slit (Fig. 1). Using the terminology of crystallography, the direction of light propagation will be a "screw direction" of the unit cell (that is to say it will have a sense of twist), if several conditions are met [22]. First, the unit cell itself shall not have an inversion center. This is assured by an asymmetry of the ring split. Second, there should be no reflection symmetry in the plane perpendicular to the propagation direction, which is provided by oblique incidence. Third, there should be no inversion or mirror rotation axis along the propagation direction. This is provided by oblique incidence and the asymmetric split. And finally there should be no reflection symmetry for any plane containing the propagation
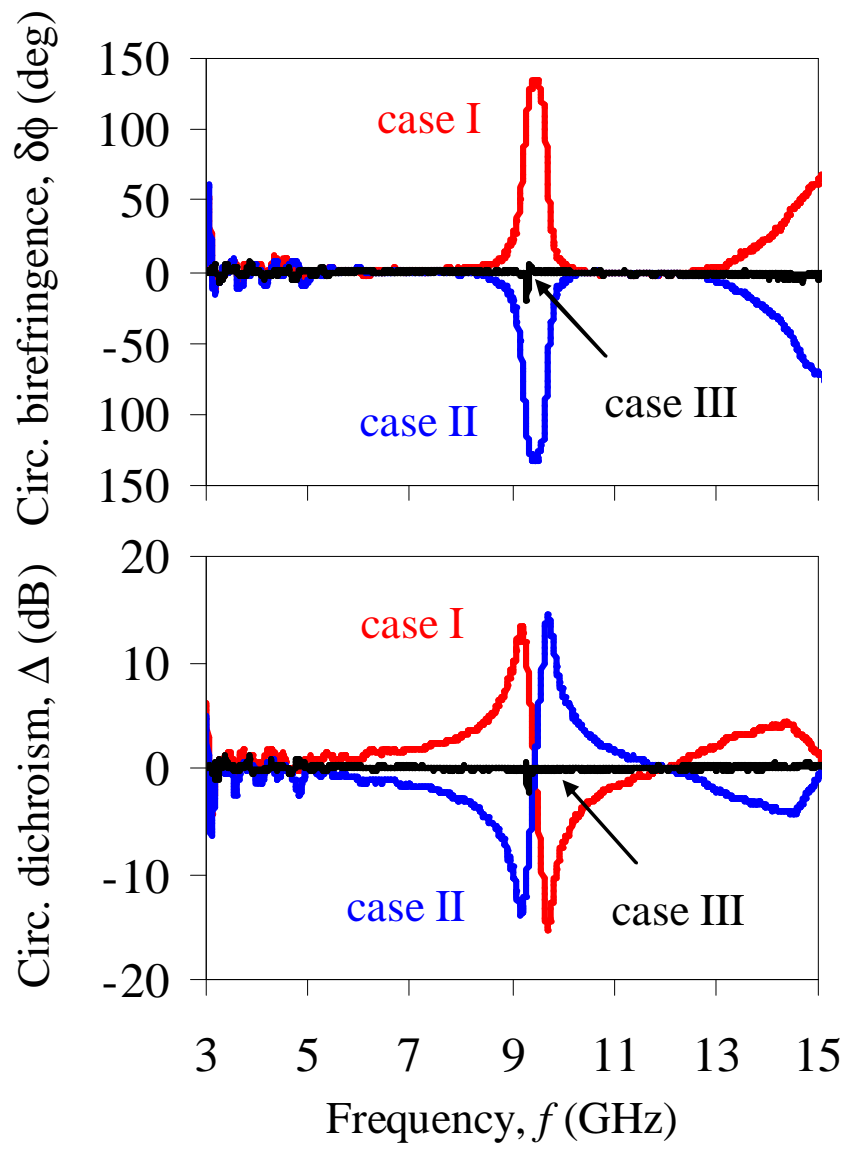

FIG. 3: (Color online) (a) Circular dichroism $\Delta$ and (b) circular birefringence $\delta \phi$ of the planar metamaterial structure measured for transmitted waves at incidence conditions I, II (tilt angle $\left.\alpha= \pm 30^{\circ}\right)$ and III $\left(\alpha=0^{\circ}\right)$ shown in Fig. 1.

direction. This requirement is only fulfilled if the split is not perpendicular, and therefore vector $s$ is not parallel, to the incidence plane $y z$. Therefore, with reference to Fig. 1, in cases I and II the direction of light propagation is a screw direction and supports optical activity. On the contrary, case III, i.e. normal incidence, fails the second, third and forth conditions of the "screw direction" test. For instance at normal incidence there is a plane of reflection symmetry containing the propagation direction.

We observed optical activity in a self-standing thin metal plate perforated with a regular two-dimensional array of ring slits (Fig. 2). The ring slits are split asymmetrically into pairs of arcs of different length separated by equal gaps. Each split ring has a line of mirror symmetry along the $x$-axis but has no axis of two-fold rotation, which enables the introduction of a polar vector $s$ that points in our case towards the short arc (see Fig. 1). The planar metamaterial is approximately $220 \times 220 \mathrm{~mm}^{2}$ in size and it has a square unit cell of $15 \times 15 \mathrm{~mm}^{2}$, which ensures that the structure does not diffract electromagnetic radiation at normal incidence for frequencies lower than $20 \mathrm{GHz}$. Our measurements were performed in an 
anechoic chamber in the $3-15 \mathrm{GHz}$ range of frequencies using broadband horn antennas (Schwarzbeck BBHA 9120D) equipped with lens concentrators and a vector network analyzer (Agilent E8364B). We measured losses and phase delays for circularly polarized electromagnetic waves transmitted by the metamaterial (see Fig. 3).

The slit structure has a number of intriguing and useful properties. Being essentially a perforated sheet of metal, it is not transparent to electromagnetic radiation apart from a narrow spectral range around the resonant frequency, at which the wavelength is approximately twice the length of the slits. Transmission at the resonance is "extraordinarily" high and substantially exceeds that given by the fraction of the area taken by the slits. As Joule losses in metals at these frequencies are negligible, the incident energy is split between reflected and transmitted radiation, and at the resonance reflection is low. Around the resonance frequency and up to one octave above it the structure does not diffract electromagnetic radiation: it becomes diffractive for wavelengths shorter than the pitch of the array. As will be illustrated below the structure shows a strong bell-shaped resonance of circular birefringence leading to strong polarization rotation, while circular dichroism is zero in the resonance. This very useful feature is in striking contrast with optical activity in most molecular systems, where characteristically strong resonant polarization rotation is accompanied by substantial circular dichroism resulting in elliptical polarization. Moreover at the optical activity resonance the system shows no linear birefringence (anisotropy) and eigenstates are therefore two circular polarizations with equally moderate losses.

If the structure is considered as a "black box" the measurements of losses and phase delays for circularly polarized electromagnetic waves provide information on the circular dichroism and optical activity of the medium in the "black box". In practical terms we measured the complex transmission matrix $t_{i j}$ for circularly polarized waves, where subscripts + and - denote right and left circularly polarized waves correspondingly. Our measurements show that the diagonal elements $\left(t_{++}\right.$and $\left.t_{--}\right)$ are generally not equal indicating that the structure has true optical activity. The difference between magnitudes of the diagonal elements $\Delta=\left|t_{++}\right|^{2}-\left|t_{--}\right|^{2}$ is a measure of circular dichroism of the "black box", while the corresponding phase difference $\delta \phi=\arg \left(t_{++}\right)-\arg \left(t_{--}\right)$ is a measure of its circular birefringence (see Fig. 3 ). The off-diagonal elements of the matrix are equal within experiential accuracy, which indicates the expected presence of some linear anisotropy in the structure but also shows a complete absence of the asymmetric transmission effect recently discovered in planar chiral structures [21]. Importantly the structure's gyrotropic properties cannot by explained by linear anisotropy, which does not contribute to $\Delta$ and $\delta$. Particularly, while linear anisotropy causes a polarization state dependent modulation of az-

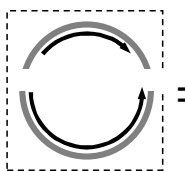

(a)

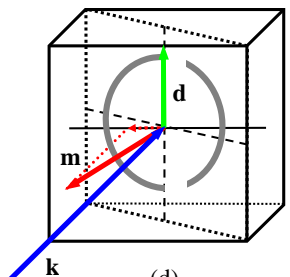

(d)

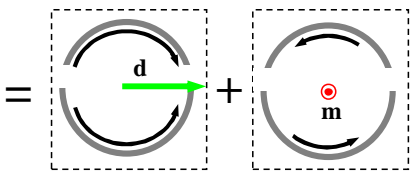

(b)

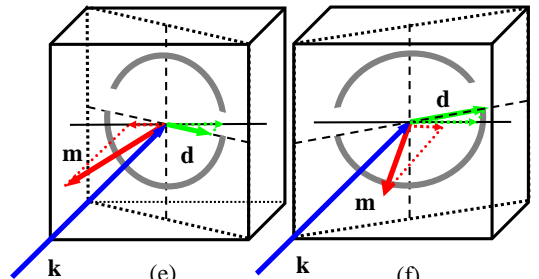

(e)

(f)
FIG. 4: (Color online) Electric and magnetic responses in an asymmetrically split wire ring. Oscillating currents in the split ring (a) can be represented as a sum of symmetric (b) and anti-symmetric (c) currents that correspond to the induced electric dipole in the plane of the ring $d$ (green arrow) and magnetic dipole perpendicular to the plane $m$ (red arrow). For tilted asymmetrically split rings polarization rotation is only absent if the projections of $d$ and $m$ onto the plane perpendicular to the $k$-vector (correspondingly green and red dashed arrows) are orthogonal (d). If these projections are either parallel (e) or anti-parallel (f), the strongest polarization rotation occurs, where the optical activity for cases (e) and (f) has opposite signs.

imuth rotation, it has no effect on the material's average polarization rotary power, which is only determined by $\delta$. In all cases experiments performed in opposite directions of wave propagation show identical results.

The following characteristic features of the effect have been observed in the experiments: i) no circular birefringence or dichroism is seen at normal incidence to the metamaterial array $(\alpha=0)$; ii) equal tilt in opposite directions yields circular dichroism and circular birefringence of opposite sign. The observed effect has a resonant nature. It is strongest around the resonance between $9 \mathrm{GHz}$ and $10 \mathrm{GHz}$, where the average arc length corresponds to approximately half the wavelength.

The microscopic origin of optical activity of the slit metamaterial the can be easily understood by considering the complementary structure, i.e. not the array of slits, but an array of metal wires in the form of split rings (see Fig. 4). As with conventional optical activity exhibited by chiral molecules, the effect must result from the presence of both electric and magnetic responses. Here, the structural asymmetry of the split rings plays a key role: as illustrated in Fig. 4(a) a wave polarized along the split induces unequal oscillating currents in the upper and lower arches of the ring. This may be represented as a sum of symmetric and antisymmetric currents that correspond to the induced electric dipole in the plane of the ring and magnetic dipole perpendicular to the ring (see Figs. 4(b) and (c)). 


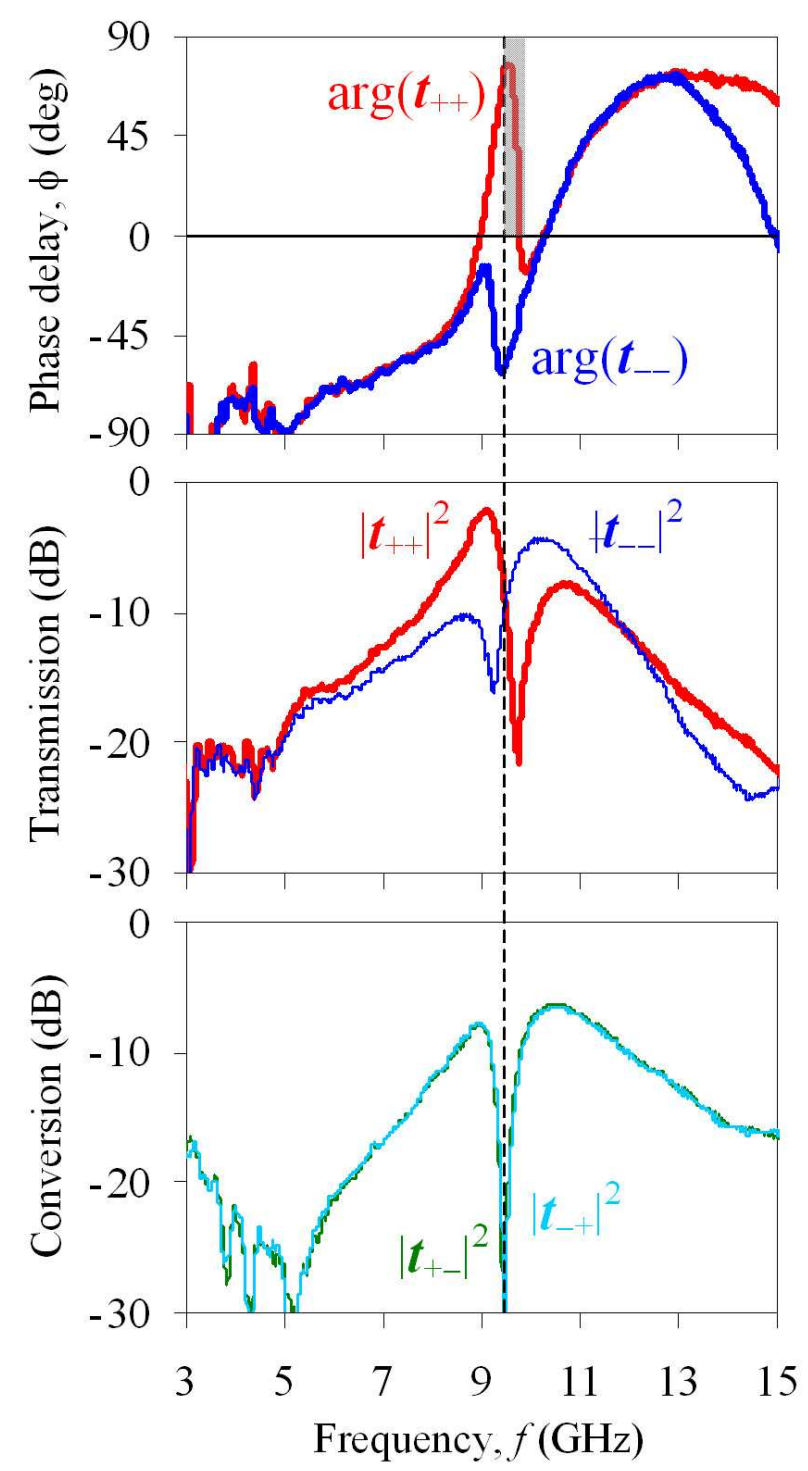

FIG. 5: (Color online) (a) Dispersions of phase delay $\phi$ for the transmitted left and right circularly polarized waves. The shaded area indicates the frequency range with almost circular eigenstates, where the phase velocity $v_{p}$ and group velocity $v_{g}$ for right circular polarization have opposite signs. (b) Transmitted intensity of both left and right circularly polarized waves. (c) The efficiency of circular polarization conversion, which is a direct indication of anisotropy (linear birefringence) of the metamaterial response. The data in all panels correspond to incidence condition I shown in Fig. 1, where the tilt angle is $\alpha=30^{\circ}$.

Now we shall consider non-normal incidence of the wave on the structure (see Figs. 4(d)-4(f)). Here blue, red and green arrows represent the wave vector $k$ and induced magnetic $m$ and electric $d$ dipoles of the metamaterial's unit cell, while dashed arrows show projections of the corresponding dipole moments onto the plane perpendicular to the wave vector. The structure shows optical activity if the split is not perpendicular to the plane of incidence. Maximum optical activity is observed when the split is parallel to the plane of incidence, in this case the wave vector and induced magnetic and electric dipoles are coplanar. The mutual phase difference between the electric and magnetic responses and thus the sign of optical activity depends on the sign of the tilt (compare projections of electric and magnetic dipoles in Fig. 4(e) and $4(\mathrm{f})$ ). Similarly to how it happens in conventional chiral media, when the wave vector and induced magnetic and electric dipoles of the "meta-molecule" are coplanar, the oscillating dipole components perpendicular to the $k$-vector create scattered electromagnetic waves with orthogonal polarizations in the direction of wave propagation, and therefore the polarization of the transmitted wave rotates. On the contrary, if the split is perpendicular to the plane of incidence, the induced magnetic and electric dipoles as well as their projections are orthogonal and the structure does not show any optical activity (see Fig. 4(d)): the oscillating magnetic and electric dipoles emit electromagnetic waves of the same polarization that propagate along the direction of the incident wave. According to Babinet's principle the slit metamaterial structure that is complementary to the wire structure discussed above, will exhibit similar polarization resonances in the same frequency band.

Intriguingly, in the slit metamaterial, at the resonance spectral band from about 9 to $10 \mathrm{GHz}$, phase velocity $\left(v_{p} \sim \omega / \phi\right.$, where $\left.\omega=2 \pi f\right)$ and group velocity $\left(v_{g} \sim d \omega / d \phi\right)$ for right circular polarization have opposite signs indicating the appearance of a backward wave (see Fig. 5(a)). In accordance with Pendry [1] this is a necessary condition or signature of negative refraction in bulk chiral media. Following Pendry negative refraction should be seen at the resonance for one circular polarization only swapping to the other one in the enantiomeric form of the medium. In agreement with this, our experiments show opposite signs of group and phase velocities for right circular polarization at $\alpha=30^{\circ}$ and for left circularly polarized waves for the enantiomeric arrangement, at $\alpha=-30^{\circ}$. Importantly, linear anisotropy essentially disappears (here circular conversion $t_{+-}=t_{-+}=\frac{1}{2} \cdot\left(t_{x x}-t_{y y}\right)$ is negligible, as shown in Fig. 5(c)). Thus the polarization eigenstates are very close to circular and in the $k$-vector direction the material behaves as isotropic optically active medium. Moreover, in this spectral range losses represented by $\left|t_{++}\right|^{2}$ and $\left|t_{--}\right|^{2}$ are relatively small (see Fig. 5(b)). Our results indicate that there may be an opportunity to develop novel negative index media for circular polarization based on simple achiral building blocks, where negative refraction would arise from exceptionally large optical activity.

In conclusion we have demonstrated strong resonant optical activity and circular dichroism using a non-chiral planar metamaterial. We have shown that for artificial structures chirality arising from the mutual orientation 
of a non-chiral structure and the incident beam can be sufficient to lead to an exceptionally strong gyrotropic response.

Financial support of the Engineering and Physical Sciences Research Council, UK is acknowledged.

* Email: n.i.zheludev@soton.ac.uk; Homepage:www . nanophotonics.org.uk/niz/

[1] J. B. Pendry, Science 306, 1353 (2004).

[2] S. Tretyakov, A. Sihvola, and L. Jylha, Photonics Nanostruct. Fundam. Appl. 3, 107 (2005).

[3] C. Monzon and D. W. Forester, Phys. Rev. Lett. 95, 123904 (2005).

[4] Y. Jin and S. He, Opt. Express 13, 4974 (2005).

[5] Q. Cheng and T. J. Cui, Phys. Rev. B 73, 113104 (2006).

[6] V. M. Agranovich, Y. N. Gartstein, and A. A. Zakhidov, Phys. Rev. B 73, 045114 (2006).

[7] J. B. Pendry, Phys. Rev. Lett. 85, 3966 (2000).

[8] A. V. Rogacheva, V. A. Fedotov, A. S. Schwanecke, and N. I. Zheludev, Phys. Rev. Lett. 97, 177401 (2006).

[9] M. D. M. Klein, M. Wegener, and S. Linden, Opt. Lett. 32, 856 (2007).

[10] K. Konishi, T. Sugimoto, B. Bai, Y. Svirko, and
M. Kuwata-Gonokami, Opt. Express 15, 9575 (2007).

[11] E. Plum, V. A. Fedotov, A. S. Schwanecke, N. I. Zheludev, and Y. Chen, Appl. Phys. Lett. 90, 223113 (2007).

[12] M. Thiel, M. Decker, M. Deubel, M. Wegener, and S. L. G. von Freymann, Adv. Mat. 19, 207 (2007).

[13] E. Plum, J. Dong, J. Zhou, V. A. Fedotov, T. Koschny, C. M. Soukoulis, and N. I. Zheludev, arXiv:0806.0823v1 (2008).

[14] I. Tinoco and M. P. Freeman, J. Phys. Chem. 61, 1196 (1957).

[15] D. L. Jaggard and N. Engheta, Electron. Lett. 25, 173 (1989).

[16] F. Mariotte and N. Engheta, Radio Sci. 35, 827 (1995).

[17] M. W. Horn, M. D. Pickett, R. Messier, and A. Lakhtakia, Nanotech. 15, 303 (2004).

[18] C. W. Bunn, Chemical Crystallography (Oxford University Press, New York, 1945), p. 88.

[19] R. Williams, Phys. Rev. Lett. 21, 342 (1968).

[20] A. Papakostas, A. Potts, D. M. Bagnall, S. I. Prosvirnin, H. J. Coles, and N. I. Zheludev, Phys. Rev. Lett. 90, 107404 (2003).

[21] V. A. Fedotov, P. L. Mladyonov, S. L. Prosvirnin, A. V. Rogacheva, Y. Chen, and N. I. Zheludev, Phys. Rev. Lett. 97, 167401 (2006).

[22] I. I. Sirotin and M. P. Shaskolskaya, Crystal physics (Nauka, Moscow, 1975), in Russian. 